\title{
Latest results of Skyrme-Hartree-Fock-Bogoliubov mass formulas
}

\author{
S. Goriely ${ }^{1, a}$ and J.M. Pearson ${ }^{2}$ \\ ${ }^{1}$ Institut d'Astronomie et d'Astrophysique, Université Libre de Bruxelles, Campus de la Plaine CP 226, 1050 Brussels, Belgium \\ 2 Dépt. de Physique, Université de Montréal, Montréal (Québec), H3C 3J7 Canada
}

\begin{abstract}
The latest developments made in deriving accurate mass predictions within the microscopic Skyrme Hartree-Fock-Bogolyubov (HFB) approach are described. In particular, all our previous HFB mass models up to and including HFB-9 suffered from the defect of excessively strong pairing, as a result of the pairing parameters being fitted freely to the mass data. Within the same HFB framework, and maintaining the condition that neutron matter be fitted, we construct new models in which the pairing is constrained in such a way as to bring down the spectral pairing gap to be close to the experimental even-odd differences. The corresponding HFB model is shown to provide more reliable input for nuclear level density calculation. In addition, a renewed attention has been paid to an accurate determination of fission barriers on the basis of the same HFB approach. It is shown that with the introduction of a phenomenological vibrational correction, a good agreement with experimental fission barriers can be obtained without deteriorating the mass fit.
\end{abstract}

\section{Introduction}

In the last few years it has become possible to construct complete mass tables by the Hartree-Fock (HF) method [1-10], with the parameters of the underlying forces being fitted to essentially all of the available mass data. These HF calculations are based on conventional Skyrme forces. A $\delta$-function pairing force acting between like nucleons is also included $n$ the full Bogoliubov framework (HFB), as well as a phenomenological Wigner term (see [8] for more details). The models HFB-1 to HFB-7 [3-6] are characterized by different treatments of the pairing force and of the effective nucleon mass, while in the case of HFB-8 and HFB-9 [7,8] we project with the PLN (projected Lipkin-Nogami) method states of good particle number. However, despite the differences between them, models HFB-2 to HFB-8 all give comparably good fits to the mass data: the rms deviation with respect to the 2149 measured masses of nuclei with $N$ and $Z \geq 8$ given in the 2003 Atomic Mass Evaluation (AME) [11] is in every case less than $0.7 \mathrm{MeV}$, the best being HFB-8, for which the rms deviation is $0.635 \mathrm{MeV}$.

Since the publication of HFB- 8 , we have generated several new mass models, but the direction of our work has shifted. Rather than seek ever better fits to the mass data our concern has been more with the construction of a universal force for all the various astrophysical applications, and to this end we have been imposing on our mass models extra physical constraints. Here it is important to recall that for practical applications, mass models are expected to provide not only $Q$-values for reaction cross section calculations but also all the nuclear structure properties required in such calculations. These include deformation parameters, single-particle level schemes, radii, neutron and proton densities, shell and pairing energies. All these nuclear ingredients are necessary to estimate the nuclear level densities, $\gamma$-ray strength functions and optical model potentials. In addition to the ground state

${ }^{a}$ Presenting author, e-mail: sgoriely@astro.ulb.ac.be properties, the calculation of fission probabilities also requires spanning the full deformation space for a reliable determination of fission paths, barrier heights and widths, as well as inertial mass parameters and nuclear level densities along the fission path. For these reasons, in the new models developed since the publication of HFB-8 [7], we have focussed on either constraining parameters to additional observables or improving the description of some correlation energies. More specifically, our latest models [8-10], presented in section 2, were subjected to the following constraints:

i) The energy-density curve of neutron matter was fitted, a requirement that is relevant not only to neutron-star applications, but also to the reliability of finite-nucleus extra polations out towards the neutron drip line. The properties of neutron matter cannot, of course, be measured in the laboratory, but they can be calculated with a high degree of reliability from realistic two- and three-nucleon forces; we took as the basis for our mass fit the zero-temperature results of Friedman and Pandharipande (FP) [12]. For all mass models from HFB-9 to HFB-14, an excellent fit to the FP curve was achieved simply by imposing a nuclearmatter symmetry coefficient of $J=30 \mathrm{MeV}$.

ii) The pairing force for the HFB-10 to HFB-14 models was considerably weakened with respect to that of all our previous mass models $[9,10]$, for which the pairing was excessively strong, in the sense that despite their good mass fits the calculated spectral pairing gaps were much bigger than the experimental even-odd mass differences. The pairing strength plays a decisive role for a reliable predictions of properties like nuclear level densities or fission barriers, as will be shown in section 3 .

iii) A more general form of collective (rotational plus vibrational) correction was introduced and fitted to experimental energies of shape isomers and fission barriers. All forces prior to HFB-14 (which only consider a rotational correction based on the cranking model) turn out to poorly predict the $Z<92$ primary barriers. Fitting a model to nuclear masses does not explore all the regions of 


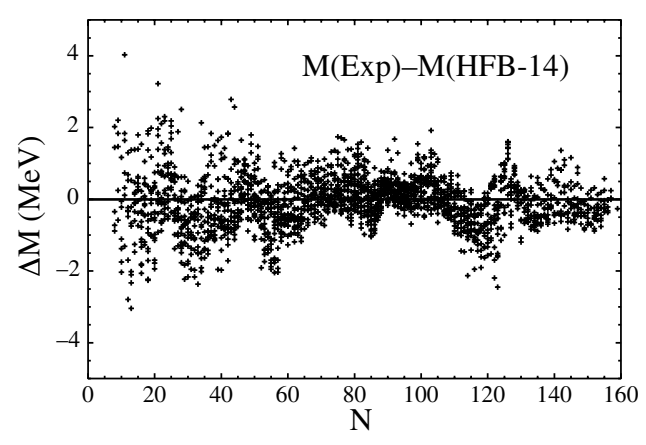

Fig. 1. Differences between experimental and calculated masses as a function of the neutron number $N$ for the HFB-14 mass model.

deformation space that are relevant to barriers. However, it is possible, through adjustment of a phenomenological vibrational term that we have included for the first time in our HFB-14 model [10] to obtain drastically improved barriers without any deterioration of the mass fit. These will be presented in section 4 .

Despite the imposition of these conditions, the quality of the mass fit was only slightly worse with HFB-9 to HFB-14 than with HFB-8, the rms deviation rising to acceptable values in the range 0.720 to $0.755 \mathrm{MeV}$ for the same data set. The HFB-14 mass predictions are rapidly described in section 2 and the advantages of this new mass model over our earlier ones are discussed in sections 3 and 4.

\section{HFB-14 mass model}

As explained in section 1, the HFB-14 mass model represents our latest attempt to provide a universal force capable of predicting globally static nuclear structure properties. The deviations between the 2149 measured masses of nuclei with $N$ and $Z \geq 8$ given in the 2003 AME [11] and the HFB-14 predictions are shown graphically in figure 1 . The rms and mean (data - theory) values of these deviations are $0.729 \mathrm{MeV}$ and $-0.057 \mathrm{MeV}$, respectively. Even if the rms deviation is about $100 \mathrm{keV}$ larger than the one obtained with our best-fit HFB-8 mass model, HFB-14 satisfies extra physical constraints that make it more suitable for the calculation of reaction cross sections in particular. Our calculated quadrupole moments, charge radii and charge-density distributions are in excellent agreement with experiment.

In figure 2 we compare the HFB-14 predictions with those of the finite range droplet model (FRDM) [13], plotting the differences as a function of both the neutron number $N$ and the neutron-separation energy $S_{n}$ for all $8 \leq Z \leq 110$ nuclei lying between the proton and neutron drip lines. Significant differences are found, especially for heavy neutron-rich nuclei. Such differences are much larger than what is obtained between the various HFB mass models [10].

\section{HFB mass models and nuclear level densities}

One drawback with all our HFB calculations prior to [9] lies in the effective interaction which is characterized by an

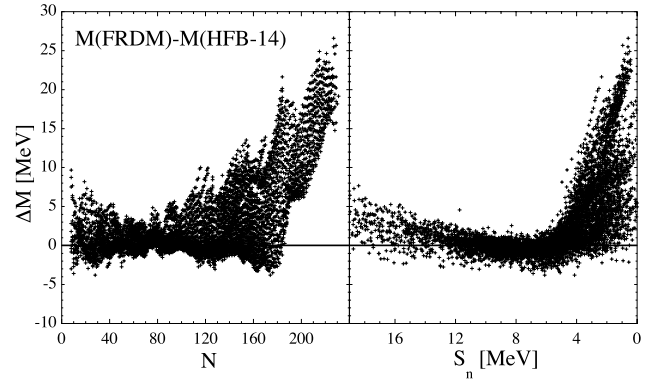

Fig. 2. Differences between the FRDM and HFB-14 mass predictions as a function of (left) $N$ and (right) $S_{n}$ for all $8 \leq Z \leq 110$ nuclei lying between the proton and neutron drip lines.

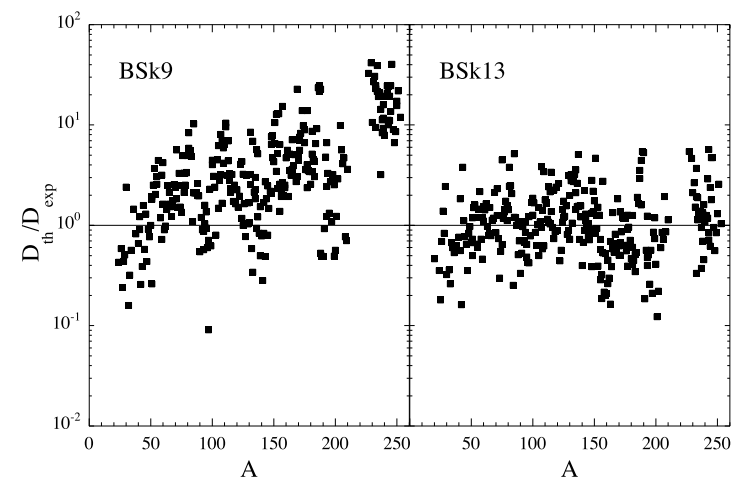

Fig. 3. Left panel: Ratio of theoretical $\left(D_{t h}\right)$ HFB plus combinatorial model based on the BSk9 Skyrme force to experimental $\left(D_{\text {exp }}\right)$ s-wave neutron resonance spacings for the 295 nuclei compiled in [17]. Right panel: Same for the BSk13 force.

excessive strength of the pairing force. If the pairing force is fitted directly to even-odd mass differences then open-shell spherical nuclei tend to be badly underbound. The most obvious origin of this deficiency is the failure to go beyond the mean-field approximation and take explicit account of vibrational correlations [14]. The fact that such a pairing force is always stronger than is required by the even-odd mass differences manifestly favours the quality of the fits to the mass data. However, such a strong pairing has an extremely detrimental impact on the prediction of nuclear level densities.

The HFB-10 to HFB-14 mass models have been constructed with a strongly reduced pairing strength of the effective interaction, while maintaining the quality of the mass fit [9]. While HFB-10 to HFB-14 differ in the HFB framework used (in particular, HFB-10 includes particle projection, while HFB-11 to HFB-13 differ in the pairing cut-off and the possible regularization of the pairing equations following the procedure of [15]), their pairing interactions were all constrained to reproduce globally the odd-even mass differences.

The strong-pairing BSk9 and weak-pairing BSk13 effective interactions have been used to generate all nuclear structure properties, and most particularly the single-particle level schemes in the equilibrium configuration, including the pairing strength for each single-particle level needed to estimate the nuclear level density within the combinatorial approach [16]. The predicted s-wave neutron spacings at the neutron separation energy $S_{n}$ are compared in figure 3 with experimental data [17]. Although the BSk9 interaction is 


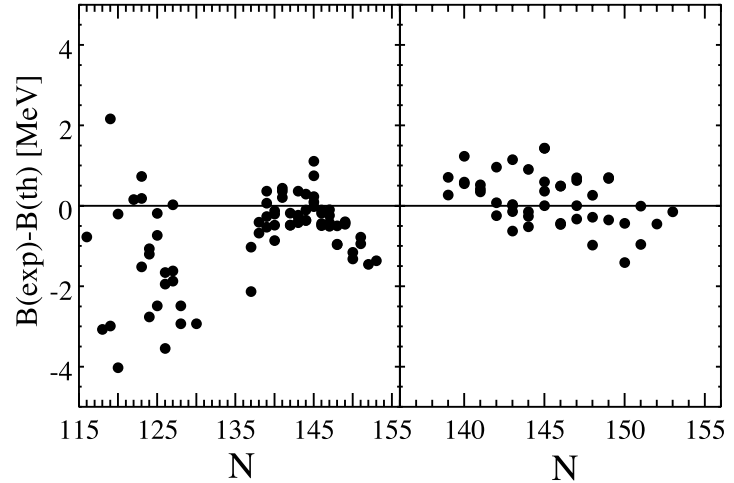

Fig. 4. Deviations between model HFB-14 and experiment for a) upper panel: primary barriers; b) middle panel: secondary barriers; c) lower panel: isomeric states.

globally similar to BSk13, the major difference lying in the pairing strength, there is a clear disagreement when using the BSk9 nuclear input, deviations up to a factor of 53 being found. In particular, in the actinide region, the nuclear level densities are largely underestimated due to the significant pairing effect at $S_{n}$. A much more satisfactory result is obtained with the low-pairing BSk13 effective force. This comparison reflects the well-known remarkable sensitivity of nuclear level densities to the pairing strength at low energies and the importance of considering this quantity when fitting the effective force.

\section{HFB mass models and fission properties}

On the basis of the mass models prior to HFB-9, all the measured fission barriers of nuclei with $Z \geq 80$ were calculated [18]. In particular for the HFB-8 model, for nuclei with $Z \geq 92$ the rms deviation of the calculated heights of the primary barriers from the experimental values was only $0.72 \mathrm{MeV}$, but the results were much poorer for the primary barriers of nuclei with $Z<92$ : they were never less than $1.1 \mathrm{MeV}$ too high and could be as much as $5.7 \mathrm{MeV}$ too high, a much worse performance than that of the ETFSI method [19]. The sudden deterioration in the HFB primary barriers of ref. [18] as we pass from $Z=92$ to $Z=91$ was found to be correlated with a rapidly growing third barrier lying at very large deformation. All our mass models up to HFB-13 show the same pathologies for the $Z<92$ barriers. For this reason, a new mass model (HFB-14) was designed, essentially considering an additional phenomenological vibrational term. This new feature enabled us to obtain drastically improved barriers without any deterioration of the mass fit.

HFB-14 barriers and shape isomers are compared with experimental data [17] in figure 4, the upper panel showing the 77 primary barriers that have been measured, the middle panel the 45 secondary barriers, and the lower panel the 30 isomeric states (the data for these come from table I of ref. [7]). In particular, the rms deviation for the 52 primary barriers of nuclei with $88 \leq Z \leq 96$, which are always less than $9 \mathrm{MeV}$ high, is as low as $0.67 \mathrm{MeV}$. A similar accuracy is obtained $(0.65 \mathrm{MeV})$ for the secondary barriers, good values for which are necessary for a reliable calculation of fission probabilities.

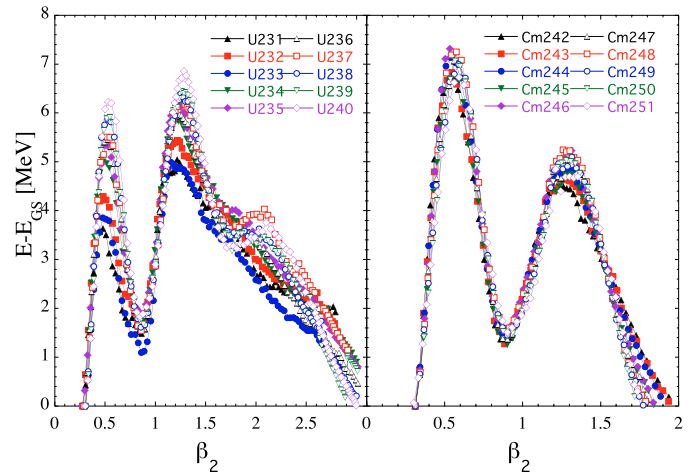

Fig. 5. Fission path (i.e., total energy with respect to the ground state energy $E_{G S}$ ) as a function of the quadrupole deformation parameter $\beta_{2}$ for $\mathrm{U}$ and $\mathrm{Cm}$ isotopes close to the valley of $\beta$ stability.

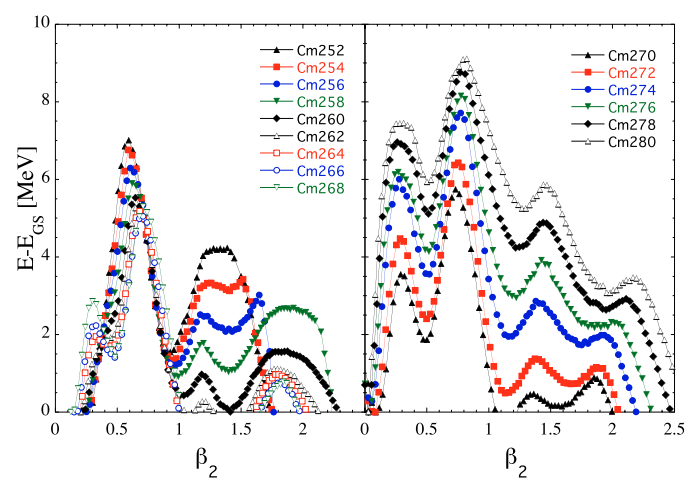

Fig. 6. Fission path as a function of the quadrupole deformation parameter $\beta_{2}$ for $\mathrm{n}$-rich even-even $\mathrm{Cm}$ isotopes.

Model HFB-14 definitely out-performs the ETFSI model [19], and is well suited for a new calculation of all the barriers involved in the r-process.

To illustrate the HFB predictions, we show in figure 5 the HFB-14 fission path for the $\mathrm{U}$ and $\mathrm{Cm}$ isotopes close to the valley of $\beta$ stability. The fission path corresponds to the most gently climbing or steepest descending path found and projected along one deformation parameter, namely the quadrupole deformation $\beta_{2}$ (for more details, see [10]). Although for the heavy isotopes of $U$ a tiny third barrier seems to appear at large deformations, the fission path for these nuclei appears to be well represented by a traditional doublehumped barrier.

The situation is however different as soon as we depart from the valley of stability. In figures 6-7, we show the fission paths for neutron-rich even- $N \mathrm{Cm}$ isotopes. For $252 \leq$ $A \leq 270$, the outer barrier located at $\beta_{2} \simeq 1.3$ disappears progressively, but reappears for $A>270$ to vanish again for $A>284$. For $A>270$, a new inner barrier at $\beta_{2} \simeq 0.3$ sets in, before vanishing again for $A \geq 286$. The primary barrier is shifted from deformation $\beta_{2} \simeq 0.6$ to values larger than 1 for the heaviest isotopes, reaching a maximum height of around $9 \mathrm{MeV}$ at the $N=184$ shell closure, and falling thereafter to around $3 \mathrm{MeV}$ for $A=288$. All these figures illustrate the complex character of the fission path for exotic nuclei and the impossibility of representing them by a traditional doublehumped path. 


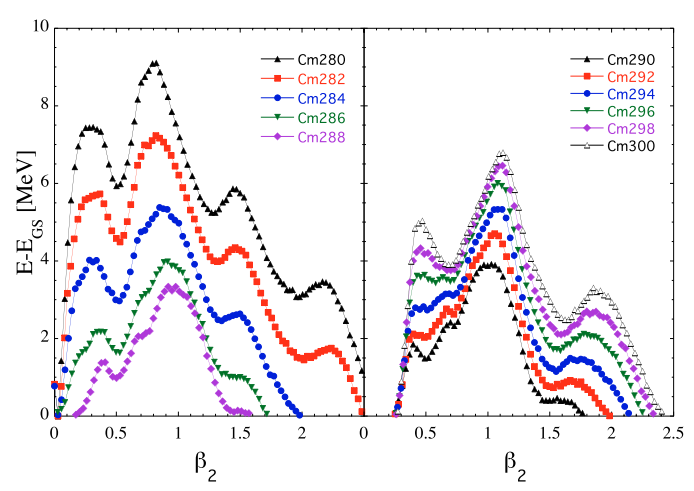

Fig. 7. Fission path as a function of the quadrupole deformation parameter $\beta_{2}$ for $\mathrm{n}$-rich even-even $\mathrm{Cm}$ isotopes.

\section{Conclusions}

A special effort has been devoted to elaborate HFB mass models which not only provide accurate mass fits, but also reproduce as many nuclear structure observables (like deformations, radii...) as possible and satisfy a maximum number of additional physical constraints, like fitting neutron matter equation of state, providing adequate input for nuclear level density calculation and reproducing at best fission barrier heights. Despite the extra physical constraints and the much better barriers, the rms error of the mass fit is not significantly worse than with our "best-fit" model HFB-8: $0.729 \mathrm{MeV}$ rather than $0.635 \mathrm{MeV}$. We believe that this slight loss in quality of the mass fit is well worth the much greater applicability of the model to nuclear applications and most particularly astrophysics problems.

S.G. is F.N.R.S senior research assistant and J.M.P. thanks the NSERC (Canada) for financial support.

\section{References}

1. F. Tondeur, S. Goriely, J.M. Pearson, M. Onsi, Phys. Rev. C 62, 024308 (2000).

2. S. Goriely, F. Tondeur, J.M. Pearson, At. Data Nucl. Data Tables 77, 311 (2001).

3. M. Samyn, S. Goriely, P.-H. Heenen, J.M. Pearson, F. Tondeur, Nucl. Phys. A 700, 142 (2002).

4. S. Goriely, M. Samyn, P.-H. Heenen, J.M. Pearson, F. Tondeur, Phys. Rev. C 66, 024326 (2002).

5. M. Samyn, S. Goriely, J.M. Pearson, Nucl. Phys. A 725, 69 (2003).

6. S. Goriely, M. Samyn, M. Bender, J.M. Pearson, Phys. Rev. C 68, 054325 (2003).

7. M. Samyn, S. Goriely, M. Bender, J.M. Pearson, Phys. Rev. C 70, 044309 (2004).

8. S. Goriely, M. Samyn, J.M. Pearson, M. Onsi, Nucl. Phys. A 750, 425 (2005).

9. S. Goriely, M. Samyn, J.M. Pearson, Nucl. Phys. A 773, 279 (2006).

10. S. Goriely, M. Samyn, J.M. Pearson, Phys. Rev. C (submitted).

11. G. Audi, A.H. Wapstra, C. Thibault, Nucl. Phys. A 729, 337 (2003).

12. B. Friedman, V.R. Pandharipande, Nucl. Phys. A 361, 502 (1981).

13. P. Möller, J.R. Nix, W.D. Myer, W.J. Swiatecki, At. Data Nucl. Data Tables 59, 185 (1995).

14. M. Bender, G.F. Bertsch, P.-H. Heenen, Phys. Rev. Lett. 94, 102503 (2005).

15. A. Bulgac, Y. Yu, Phys. Rev. Lett. 88, 042504 (2002).

16. S. Hilaire, S. Goriely, Nucl. Phys. A 779, 63 (2006).

17. Handbook for calculations of nuclear reaction data, RIPL-2 (IAEA-Tecdoc-1506, 2006).

18. M. Samyn, S. Goriely, J.M. Pearson, Phys. Rev. C 72, 044316 (2005).

19. A. Mamdouh, J.M. Pearson, M. Rayet, F. Tondeur, Nucl. Phys. A 644, 389 (1998). 